\title{
Distinct Hippocampal Expression Profiles of Long Non-coding RNAs in an Alzheimer's Disease Model
}

\author{
Bo Yang ${ }^{1} \cdot$ Zi-an Xia ${ }^{1,2} \cdot$ Bingwu Zhong $^{2} \cdot$ Xingui Xiong $^{1} \cdot$ Chenxia Sheng $^{2}$ • \\ Yang Wang ${ }^{1}$ - Wei Gong ${ }^{2}$ - Yucheng $\mathrm{Cao}^{2}$ - Zhe Wang ${ }^{2} \cdot$ Weijun Peng ${ }^{2}$ (I)
}

Received: 3 May 2016/Accepted: 3 August 2016/Published online: 8 August 2016

(C) The Author(s) 2016. This article is published with open access at Springerlink.com

\begin{abstract}
Alzheimer's disease (AD), the most prevalent form of dementia worldwide, is a complex neurodegenerative disease characterized by the progressive loss of memory and other cognitive functions. The pathogenesis of $\mathrm{AD}$ is not yet completely understood. Although long non-coding RNAs (lncRNAs) have recently been shown to play a role in $\mathrm{AD}$ pathogenesis, the specific influences of lncRNAs in AD remain largely unknown; in particular, hippocampal lncRNA expression profiles in $\mathrm{AD}$ rats are lacking. In this study, microarray analysis was performed to investigate the hippocampal expression patterns of dysregulated lncRNAs in a rat model of AD. A total of $315 \operatorname{lncRNAs}$ and 311 mRNAs were found to be significantly dysregulated in the AD model ( $\geq 2.0$ fold, $p<0.05$ ). Then, quantitative real-time PCR was used to validate the expression of selected lncRNAs and mRNAs. Bioinformatics tools and databases were employed to explore the potential lncRNA functions. This is the first study to comprehensively identify dysregulated hippocampal IncRNAs in AD and to demonstrate the involvement of different lncRNA expression patterns in the hippocampal pathogenesis of $\mathrm{AD}$. This information will enable further research
\end{abstract}

Zhe Wang and Weijun Peng contributed equally to this study.

\footnotetext{
Zhe Wang

ericwangzhe@126.com

Weijun Peng

pengweijun1987@gmail.com
}

1 Department of Integrated Traditional Chinese and Western Medicine, Xiangya Hospital, Central South University, Changsha Hunan 410008, China

2 Department of Integrated Traditional Chinese \& Western Medicine, The Second Xiangya Hospital, Central South University, Changsha, Hunan 410011, China on the pathogenesis of $\mathrm{AD}$ and facilitate the development of novel AD therapeutics targeting lncRNAs.

Keywords Alzheimer's disease $\cdot$ Long non-coding RNAs $(\operatorname{lncRNAs}) \cdot$ Microarray $\cdot$ Hippocampus $\cdot$ Expression profiles

\section{Introduction}

Alzheimer's disease (AD) is considered to be the most common cause of dementia; $\mathrm{AD}$ is a progressive neurodegenerative disease characterized by the accumulation of amyloid- $\beta(A \beta)$ plaques and neurofibrillary tangles, synaptic and neuronal loss, and cognitive decline [1]. In the USA alone, an estimated 5.2 million individuals aged 65 and older have $\mathrm{AD}$, and this number is expected increase to 13.8 million by 2050 [2]. Unfortunately, no currently available therapeutic strategies for AD slow or stop the neuronal damage that causes $\mathrm{AD}$ symptoms and eventually results in death $[3,4]$. Thus, there is an urgent need for novel strategies of improving our mechanistic understanding of $\mathrm{AD}$, which could lead to the discovery of novel therapeutic targets.

Long non-coding RNAs (lncRNAs), a subclass of ncRNAs, are most commonly defined as the transcripts of more than 200 nucleotides that structurally resemble mRNAs but have no protein-coding capacity [5]. With the development of techniques to detect lncRNA, accumulating evidence indicates that IncRNAs participate in a wide variety of important biological phenomena, such as imprinting genomic loci, influencing chromosome conformation and allosterically regulating enzymatic activity [6]. Moreover, multiple lines of evidence have linked lncRNA mutations and dysregulation with diverse human diseases, ranging from different types of cancer and neurodegeneration to gynaecological diseases [7-9]. 
The role of lncRNAs in AD has attracted considerable attention. Recent studies have further confirmed the involvement of certain lncRNAs in AD [10-13]. In addition, with recent advancements in transcriptome-wide profiling, numerous AD-associated lncRNAs have been discovered. Furthermore, the dysregulation of lncRNA expression in post-mortem tissue samples from AD patients $[14,15]$ and transgenic AD animals [16] has been investigated. Despite these findings, the expression patterns, targets, and functions of lncRNAs involved in the pathogenesis of AD remain largely unknown [17]. Therefore, further research is of great importance.

In addition, as a crucial component of the medial temporal lobe memory circuit, the hippocampus is affected early in AD and displays synaptic and intraneuronal molecular remodelling against a pathological background of extracellular $A \beta$ deposition and intracellular neurofibrillary tangle formation in the early stages of AD [18]. Moreover, behavioural studies have long suggested that the hippocampus plays a critical role in learning and memory, which depend on functional and structural changes occurring in the hippocampus, such as long-term potentiation and synaptic remodelling [19].

Therefore, in the present study, we applied microarray technology to analyse the expression profiles of lncRNAs and messenger RNAs (mRNAs) in the hippocampus of rats in a validated AD model. Additionally, gene ontology (GO) and Kyoto Encyclopedia of Genes and Genomes (KEGG) analyses were performed to predict the biological roles and potential signalling pathways of these differentially expressed lncRNAs. Moreover, an lncRNA-mRNA network analysis was conducted to further explore the potential roles of differentially expressed lncRNAs in AD pathogenesis.

\section{Materials and Methods}

\section{Ethics Statement}

All animal protocols were approved by the Central South University (Changsha, China) and were performed in compliance with the National Institutes of Health Guide for the Care and Use of Laboratory Animals. This investigation was conducted in accordance with ethical standards and the Declaration of Helsinki, as well as according to national and international guidelines. This research was approved by the authors' institutional review board.

\section{Animals and Experimental Design}

A total of 20 adult, male Sprague-Dawley rats $(250 \pm 30 \mathrm{~g})$ were purchased from the Laboratory Animal Centre of Central South University. The animals were housed under controlled conditions (12-h light/dark cycle, $25^{\circ} \mathrm{C}, 50 \pm 10 \%$ relative humidity) with water and food pellets available ad libitum.

The animals were randomly divided into the following two groups ( $n=10$ in each group): (1) the AD group and (2) the control group.

\section{Surgery}

In this experiment, we utilized an intracerebroventricular (ICV) injection of A $\beta 1-42$ oligomers into the cerebral ventricles of the animals to induce a validated AD model, as we have previously described [20]. In brief, the animals were anaesthetised with $10 \%$ chloral hydrate $(4 \mathrm{ml} / \mathrm{kg})$ and placed in a stereotactic frame. The $A \beta_{1-42}$ oligomers $(5 \mu 1 * 2$, Sigma, St. Louis, MO, USA) were injected bilaterally into the lateral ventricles through a stainless steel cannula by using the following coordinates: $1.1 \mathrm{~mm}$ posterior to the bregma, $2.2 \mathrm{~mm}$ lateral to the sagittal suture, and $3.0 \mathrm{~mm}$ beneath the dura. For the control group, the vehicle was injected bilaterally into the lateral ventricles through a stainless steel cannula by using the above-mentioned coordinates.

\section{Sample Collection}

After the Morris water maze test was completed, all rats were anaesthetised with chloral hydrate and were sacrificed via decapitation. Their hippocampal tissues were stored in liquid nitrogen followed by storage at $-80{ }^{\circ} \mathrm{C}$ prior to analysis.

\section{Morris Water Maze Test}

Spatial learning and memory deficits were evaluated using the Morris water maze test as we have previously described [21], with additional modifications. In brief, the test was conducted at 31-35 days after injury. Initial training was conducted on days 31-34 after injury; during this period, we trained the rats to locate a hidden, submerged platform using peripheral visual information. The rats were introduced into a pool at one of four entry points, and every entry point was used over the course of a day. The rats were given $60 \mathrm{~s}$ to locate the platform and were allowed to remain on the platform for $10 \mathrm{~s}$ before being removed. Rats that were unable to locate the platform within $60 \mathrm{~s}$ were placed on the platform for $10 \mathrm{~s}$ before being removed from the pool. On the 35 th day, a probe trial was conducted, in which the platform was removed and the number of crossings over the previous platform location was recorded over one 60-s trial. An overhead video camera connected to the ANY-maze video tracking system (Stoelting Co., USA) was used to record all the trials and track the movements of the animals. 


\section{RNA Extraction}

Total RNA was extracted from each hippocampal tissue sample by soaking the tissue samples in TRIzol Reagent (Invitrogen, Grand Island, NY, USA) in accordance with the manufacturer's instructions. RNA quantity and quality were measured using a Nano Drop ND-1000, and RNA integrity was assessed by standard denaturing agarose gel electrophoresis.

\section{Microarray Analysis}

Sample labelling and array hybridization were performed according to the Agilent One-Colour Microarray-Based Gene Expression Analysis protocol (Agilent Technology), with minor modifications. In brief, mRNA was purified from total RNA after the removal of rRNA (mRNA-ONLYTM Eukaryotic mRNA Isolation Kit, Epicentre). Then, each sample was amplified and transcribed into fluorescent cRNA along the entire length of the transcripts without 3 ' bias via a random priming method (Arraystar Flash RNA LabellingKit, Arraystar). The labelled cRNAs were purified using an RNeasy Mini Kit (Qiagen). The concentration and specific activity of the labelled cRNAs (pmol Cy3/ $\mu$ g cRNA) were measured using the NanoDrop ND-1000. In all, $1 \mu \mathrm{g}$ of each labelled cRNA was fragmented by adding $5 \mu$ of $10 \times$ Blocking Agent and $1 \mu$ of $25 \times$ Fragmentation Buffer and heating the mixture at $60{ }^{\circ} \mathrm{C}$ for $30 \mathrm{~min}$. Then, $25 \mu \mathrm{l}$ of $2 \times$ GE Hybridization Buffer was added to dilute the labelled cRNA. Subsequently, $50 \mu$ l of hybridization solution was dispensed into the gasket slide, which was then assembled with the lncRNA expression microarray slide. The slides were incubated for $17 \mathrm{~h}$ at $65^{\circ} \mathrm{C}$ in an Agilent Hybridization Oven. The hybridized arrays were washed, fixed and scanned using an Agilent DNA Microarray Scanner (part number G2505C). Microarray hybridization and expression data collection were performed by KangChen Bio-tech, Shanghai, China.

\section{Quantitative Real-Time-PCR Validation}

As previously described [22], total RNA was reversetranscribed into cDNA using SuperScript III Reverse Transcriptase(Invitrogen, Grand Island, NY, USA) in accordance with the manufacturer's instructions. An Applied Biosystems ViiA 7 Real-Time PCR System and $2 \times$ PCR Master Mix were used to perform quantitative real-time (qRT)-PCR (Arraystar). The reaction conditions were as follows: incubation at $95^{\circ} \mathrm{C}$ for $10 \mathrm{~min}$, followed by 40 cycles of $95^{\circ} \mathrm{C}$ for $10 \mathrm{~s}$ and $60^{\circ} \mathrm{C}$ for $1 \mathrm{~min}$. The relative lncRNA and mRNA expression levels were calculated using the $2^{-\Delta \Delta \mathrm{Ct}}$ method and were normalized to GAPDH, as an endogenous reference transcript [23]. The specific primers for each gene are listed in Table 1. The data represent the means of three experiments.

\section{GO Annotations and KEGG Pathways}

GO annotations and pathway analysis were applied to investigate the roles of all differentially expressed mRNAs, as previously described [24, 25]. In brief, GO analysis was applied to elucidate genetic regulatory networks of interest by forming hierarchical categories according to the molecular function, biological process, and cellular component aspects of the differentially expressed genes (http://www.geneontology.org). Pathway analysis was performed to explore the significant pathways of the differentially expressed genes, according to KEGG (http://www.genome.jp/kegg/).

\section{Construction of Co-Expression Network}

To identify interactions among the differentially expressed IncRNAs and mRNAs, we constructed a co-expression network based on a correlation analysis of the differentially expressed lncRNAs and mRNAs [26]. The network was constructed according to the normalized signal intensities of specific mRNA and lncRNA expression levels. Pearson's correlation coefficients equal to or greater than 0.7 were used to identify the lncRNAs and coding genes. Then, the lncRNAmRNA co-expression network was constructed using Cytoscape software (The Cytoscape Consortium, San Diego, CA, USA).

\section{Statistical Analysis}

All data are shown as the mean \pm standard error of the mean (SEM) and were analysed using the statistical software SPSS version 22.0 (SPSS Inc., Chicago, IL, USA). Student's $t$ test was performed for comparisons between two groups, whereas ANOVA was performed for repeated measures. The false discovery rate was calculated to correct the $p$ value. Differences with $p<0.05$ were considered statistically significant. Fold change (FC) and Student's $t$ test were used to analyse the statistical significance of the microarray results. FC $\geq 2$ and $p<0.05$ were considered the threshold values for designating differentially expressed lncRNAs and mRNAs.

\section{Results}

\section{Morris Water Maze Test}

Spatial learning was evaluated on days 31-34 after the rats received an ICV A $\beta_{1-42}$ injection, and a probe trial for spatial memory was conducted on day 35 . As expected, rats injected with $A \beta_{1-42}$ were less able than mice in the control group to 
Table 1 Primers designed for qRT-PCR validation of candidate lncRNAs and mRNAs

\begin{tabular}{|c|c|c|c|c|}
\hline & Forward primer & Reverse primer & $\begin{array}{l}\text { Product } \\
\text { length }\end{array}$ & $\operatorname{Tm}\left({ }^{\circ} \mathrm{C}\right)$ \\
\hline ВC092582 & $\begin{array}{l}5^{\prime} \text {-GGAGGTGAATGCTG } \\
\text { AGGAGGA-3' }\end{array}$ & $\begin{array}{l}5^{\prime} \text { - ATGAAGGTAGAGGC } \\
\text { GGTGGTC-3' }\end{array}$ & 60 & 60 \\
\hline MRAK050857 & $\begin{array}{l}5^{\prime} \text {-CCCCCAAGAACGGT } \\
\text { GGAGTG-3' }\end{array}$ & $\begin{array}{l}5^{\prime} \text {-GGAGACAGCGCCTG } \\
\text { AGAACGAG3' }\end{array}$ & 120 & 60 \\
\hline MRAK088596 & $\begin{array}{l}5^{\prime} \text {-AGGGGTAACGAACA } \\
\text { ACAAAGA-3' }\end{array}$ & $\begin{array}{l}5^{\prime} \text { - CATGGTACTCAGAA } \\
\text { TGCTAAAAT-3' }\end{array}$ & 59 & 60 \\
\hline MRAK081790 & $\begin{array}{l}5^{\prime} \text {-AAAATTGGTTGAGC } \\
\text { TGGTATAGGT-3' }\end{array}$ & $\begin{array}{l}\text { 5' -CCTTGGCATCAGTT } \\
\text { TCCTTGT-3' }\end{array}$ & 168 & 60 \\
\hline MAPK10 & $\begin{array}{l}\text { 5'-ATGTTAGTGATTGA } \\
\text { CCCAGCGAAG-3' }\end{array}$ & $\begin{array}{l}\text { 5'-TGCTCCCTTTCATC } \\
\text { CAGTTGC-3' }\end{array}$ & 60 & 63 \\
\hline S100a8 & $\begin{array}{l}\text { 5' -GGGAATCACCATGC } \\
\text { CCTCTAC-3' }\end{array}$ & $\begin{array}{l}5^{\prime} \text {-GCCCACCCTTATCA } \\
\text { CCAACAC-3' }\end{array}$ & 60 & 168 \\
\hline GAPDH & $\begin{array}{l}\text { 5' -GCTCTCTGCTCCTC } \\
\text { CCTGTTCTA-3' }\end{array}$ & $\begin{array}{l}\text { 5'-TGGTAACCAGGCGT } \\
\text { CCGATA-3' }\end{array}$ & 60 & 124 \\
\hline
\end{tabular}

find the platform and learn its location by the 34th day of training, indicating poor learning performance (Fig. 1b). In addition, compared with animals in the control group, the $\mathrm{A} \beta_{1-42}$-treated animals performed poorly and failed to recollect the location of the submerged platform on the 35 th day, demonstrated by significantly fewer crossings over the previous platform location (Fig. 1c).

\section{IncRNA and mRNA Expression Profiles in AD}

Microarray analysis was used to assess the expression levels of lncRNAs in AD rats relative to those in control rats. We identified 315 significantly dysregulated lncRNAs in the AD rats: 238 were up-regulated, while 77 were down-regulated $(\geq 2.0$ fold, $p<0.05)$. The top 40 most significantly a

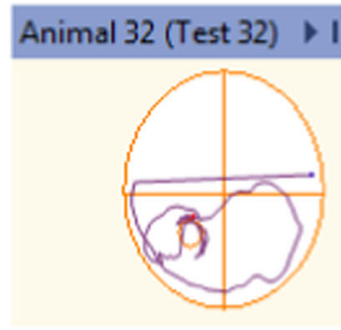

Control

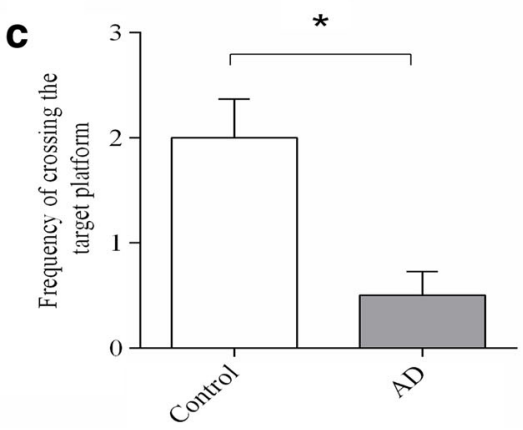

Fig. 1 Spatial learning and memory was assessed in Morris water maze test. a Representative images of the swim paths on day 34 , showing that rats in the control group were able to find the hidden platform more easily than the $\mathrm{AD}$ rats and indicating that the rats in the $\mathrm{AD}$ group (31 s) required a longer time to find the hidden platform than the rats in the control group $(15 \mathrm{~s})$; these results suggested that $A \beta_{1-42}$ impaired spatial learning ability. b Escape latency. A significant difference was detected between the control and AD groups on days 32-34 $(* p<0.05)$. $\mathbf{c}$ Probe test. The frequency of crossing the target platform was recorded as an

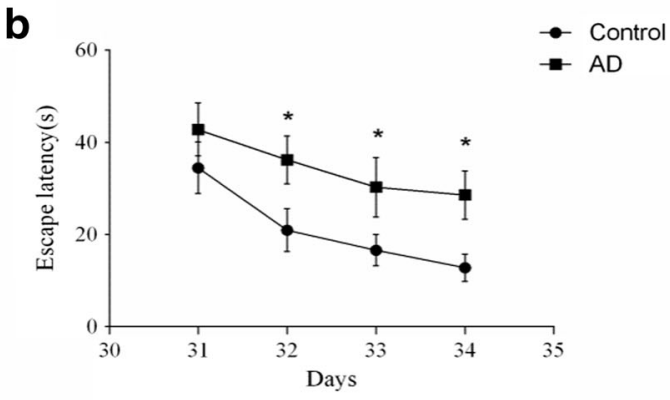

d Animal 58 (Test 58) $>$ II Animal 66 (Test 66)

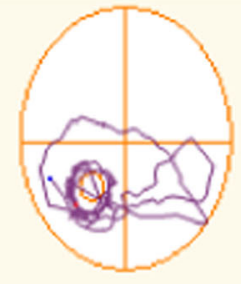

Control

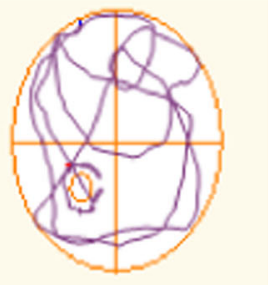

$\mathrm{AD}$ indicator of spatial memory. Significant differences were observed between the control and AD groups $(* p<0.05)$. d Representative images of the frequency of crossing the target platform, showing that rats in the control group had a crossing frequency of approximately 3 times, while that of rats in the AD group was reduced to approximately 1 time, suggesting that there was significantly better performance in the control group compared with the $\mathrm{AD}$ group; $n=6$ rats/group. Data are expressed as the mean \pm standard error of the mean (SEM) 
Table 2 Top 40 aberrantly expressed lncRNAs in microarray analysis

\begin{tabular}{|c|c|c|c|c|c|c|c|c|c|c|c|c|}
\hline SeqID & $P$ value & $\begin{array}{l}\text { Fold } \\
\text { change }\end{array}$ & Regulation & Chr & Strand & Relationship & AD-1 & AD-2 & AD-3 & Control-1 & Control-2 & Control-3 \\
\hline BC158567 & 0.01001 & 4.58596 & Down & 20 & + & $\begin{array}{l}\text { sense_intron } \\
\text { overlap }\end{array}$ & 8.00638 & 6.43733 & 7.03962 & 9.36119 & 9.59565 & 9.11816 \\
\hline BC158567 & 0.01001 & 4.58596 & Down & 20 & + & $\begin{array}{c}\text { sense_exon } \\
\text { overlap }\end{array}$ & 8.00638 & 6.43733 & 7.03962 & 9.36119 & 9.59565 & 9.11816 \\
\hline MRAK050857 & 0.00001 & 7.65238 & Down & 10 & - & others & 5.53087 & 5.65205 & 5.90255 & 8.58667 & 8.61557 & 8.69096 \\
\hline MRAK033976 & 0.00288 & 5.04632 & Down & $\mathrm{X}$ & - & others & 5.29233 & 6.53179 & 5.94278 & 8.28533 & 8.20126 & 8.28601 \\
\hline MRuc009dux & 0.00330 & 6.00053 & Down & 4 & + & $\begin{array}{l}\text { sense_intron } \\
\text { overlap }\end{array}$ & 6.03729 & 6.69110 & 6.14928 & 8.93886 & 9.46715 & 8.22693 \\
\hline MRuc009dux & 0.00330 & 6.00053 & Down & 4 & + & $\begin{array}{l}\text { antisense__ } \\
\text { intron_- } \\
\text { overlap }\end{array}$ & 6.03729 & 6.69110 & 6.14928 & 8.93886 & 9.46715 & 8.22693 \\
\hline MRAK078853 & 0.00005 & 4.46748 & Down & 4 & + & $\begin{array}{c}\text { sense_exon } \\
\text { overlap }\end{array}$ & 5.67166 & 5.69835 & 5.37772 & 7.81646 & 7.78834 & 7.62132 \\
\hline M81783 & 0.04499 & 3.88207 & Down & 6 & - & $\begin{array}{l}\text { sense_exon } \\
\text { overlap }\end{array}$ & 6.54969 & 5.91603 & 6.06684 & 8.45648 & 6.87919 & 9.06737 \\
\hline S69385 & 0.00244 & 11.25543 & Up & 8 & + & $\begin{array}{c}\text { sense_exon } \\
\text { overlap }\end{array}$ & 9.22457 & 7.80020 & 8.15135 & 4.55781 & 5.46104 & 4.67963 \\
\hline uc.28- & 0.00002 & 10.74721 & Up & 2 & + & $\begin{array}{l}\text { antisense__ } \\
\text { intron_- } \\
\text { overlap }\end{array}$ & 8.27963 & 8.00554 & 7.92272 & 4.83231 & 4.62756 & 4.47034 \\
\hline MRAK081790 & 0.00022 & 10.61600 & Up & $\mathrm{X}$ & - & $\begin{array}{l}\text { sense_intron } \\
\text { overlap }\end{array}$ & 11.47399 & 10.67374 & 10.76289 & 7.51004 & 7.73699 & 7.43908 \\
\hline XR_008107 & 0.00003 & 9.80265 & $\mathrm{Up}$ & $\mathrm{X}$ & + & others & 9.89685 & 10.08962 & 9.80230 & 6.48063 & 6.90673 & 6.52189 \\
\hline AB072252 & 0.02153 & 9.80128 & Up & 1 & - & others & 8.06770 & 6.20245 & 7.09967 & 4.11682 & 4.90890 & 2.46520 \\
\hline uc.128- & 0.01416 & 6.99610 & $\mathrm{Up}$ & 8 & + & intergenic & 7.46133 & 6.05214 & 6.17050 & 4.63293 & 3.73602 & 2.89537 \\
\hline uc. $80-$ & 0.02034 & 4.03223 & Up & 3 & - & $\begin{array}{l}\text { sense_intron } \\
\text { overlap }\end{array}$ & 4.03512 & 5.57163 & 4.80373 & 3.40618 & 2.50436 & 2.46520 \\
\hline XR_006076 & 0.00058 & 4.22020 & Up & 18 & - & others & 4.55077 & 4.92157 & 4.75323 & 3.02408 & 2.50436 & 2.46520 \\
\hline MRAK047420 & 0.00084 & 4.01561 & Up & 5 & + & $\begin{array}{l}\text { sense_intron } \\
\text { overlap }\end{array}$ & 5.46621 & 6.04554 & 5.64582 & 3.90005 & 3.43310 & 3.80757 \\
\hline XR_006113 & 0.00019 & 4.89262 & Up & 3 & + & $\begin{array}{l}\text { sense_intron } \\
\text { overlap }\end{array}$ & 12.79504 & 13.15587 & 12.89520 & 10.60371 & 10.91719 & 10.45340 \\
\hline XR_009079 & 0.00043 & 4.43645 & Up & 7 & + & others & 8.96824 & 9.31937 & 8.92314 & 6.92116 & 7.19221 & 6.64916 \\
\hline XR_006120 & 0.00028 & 3.95666 & Up & 3 & + & intergenic & 12.37788 & 12.39927 & 12.13337 & 10.29845 & 10.57616 & 10.08305 \\
\hline MRAK043605 & 0.00012 & 3.71597 & Up & 10 & + & $\begin{array}{c}\text { antisense_- } \\
\text { exon_- } \\
\text { overlap }\end{array}$ & 9.04173 & 9.15901 & 8.99951 & 7.05250 & 7.40744 & 7.05909 \\
\hline MRAK043605 & 0.00012 & 3.71597 & Up & 10 & + & $\begin{array}{c}\text { antisense_ } \\
\text { exon_- } \\
\text { overlap }\end{array}$ & 9.04173 & 9.15901 & 8.99951 & 7.05250 & 7.40744 & 7.05909 \\
\hline XR_009080 & 0.00018 & 6.67415 & Up & 1 & - & others & 11.91682 & 12.54961 & 12.23871 & 9.43643 & 9.68088 & 9.37209 \\
\hline XR_009128 & 0.00004 & 6.54726 & Up & 16 & - & intergenic & 9.47314 & 9.82283 & 9.57302 & 6.93783 & 7.06204 & 6.73645 \\
\hline MRAK158075 & 0.00013 & 6.21123 & Up & 19 & + & $\begin{array}{c}\text { antisense_ } \\
\text { exon_- } \\
\text { overlap }\end{array}$ & 11.63517 & 12.06746 & 11.72937 & 9.16909 & 9.39121 & 8.96705 \\
\hline MRuc007nww & 0.00018 & 5.77962 & Up & 6 & + & intergenic & 12.46794 & 13.04085 & 12.73809 & 10.17715 & 10.38814 & 10.08866 \\
\hline XR_008038 & 0.00008 & 5.72687 & $\mathrm{Up}$ & 2 & - & intergenic & 11.56619 & 11.93042 & 11.66727 & 9.13306 & 9.41106 & 9.06652 \\
\hline XR_007242 & 0.00001 & 7.29233 & Up & 15 & + & intergenic & 9.60316 & 9.58071 & 9.36365 & 6.59651 & 6.79242 & 6.55945 \\
\hline XR_008129 & 0.00001 & 7.07133 & Up & 16 & - & intergenic & 8.37982 & 8.26123 & 8.10604 & 5.44713 & 5.51678 & 5.31723 \\
\hline XR_008124 & 0.00034 & 6.46782 & Up & 18 & - & others & 6.60833 & 6.00739 & 5.97723 & 3.32404 & 3.46613 & 3.72295 \\
\hline XR_006337 & 0.00099 & 6.59840 & Up & 12 & - & intergenic & 6.06002 & 5.51444 & 5.50684 & 3.15227 & 3.29748 & 2.46520 \\
\hline U89530 & 0.00040 & 5.56718 & Up & 3 & - & intergenic & 6.49347 & 6.00548 & 5.95748 & 3.73959 & 3.89338 & 3.39263 \\
\hline MRAK044406 & 0.01616 & 4.85928 & $\mathrm{Up}$ & 2 & - & $\begin{array}{c}\text { sense_exon_ } \\
\text { overlap }\end{array}$ & 7.99810 & 6.33157 & 6.47274 & 4.56294 & 5.03938 & 4.35786 \\
\hline uc. $243+$ & 0.01513 & 3.76335 & Up & 2 & - & intergenic & 9.44347 & 8.51668 & 8.32754 & 6.51866 & 7.48587 & 6.54710 \\
\hline AJ131563 & 0.00182 & 4.62465 & Up & 16 & + & & 14.15639 & 13.39135 & 13.57111 & 11.38921 & 11.87005 & 11.23156 \\
\hline
\end{tabular}


Table 2 (continued)

\begin{tabular}{|c|c|c|c|c|c|c|c|c|c|c|c|c|}
\hline SeqID & $P$ value & $\begin{array}{l}\text { Fold } \\
\text { change }\end{array}$ & Regulation & Chr & Strand & Relationship & AD-1 & AD-2 & AD-3 & Control-1 & Control-2 & Control-3 \\
\hline & & & & & & $\begin{array}{c}\text { sense_exon_ } \\
\text { overlap }\end{array}$ & & & & & & \\
\hline DQ223059 & 0.00212 & 4.47442 & Up & 10 & + & others & 12.99165 & 12.36331 & 12.52671 & 10.34195 & 10.93173 & 10.12288 \\
\hline MRAK030941 & 0.00135 & 4.04066 & Up & 4 & + & $\begin{array}{c}\text { sense_exon_ } \\
\text { overlap }\end{array}$ & 15.33834 & 14.99113 & 15.12357 & 13.02945 & 13.58079 & 12.79903 \\
\hline uc. $253+$ & 0.00427 & 3.70040 & Up & 5 & + & intergenic & 11.93550 & 11.18617 & 11.56148 & 9.58592 & 10.12549 & 9.30870 \\
\hline uc. $412+$ & 0.00022 & 3.98847 & Up & 10 & - & $\begin{array}{l}\text { sense_intron } \\
\text { overlap }\end{array}$ & 4.76540 & 4.37265 & 4.24680 & 2.42778 & 2.50436 & 2.46520 \\
\hline XR_006990 & 0.00213 & 3.59883 & Up & Un & + & intergenic & 5.02968 & 4.64237 & 4.48417 & 3.01448 & 3.13395 & 2.46520 \\
\hline
\end{tabular}

SeqID: IncRNA name. $P$ value:P value calculated from unpaired t-test. Fold Change: the absolute ratio (no log scale) of normalized intensities between two groups(AD vs Control). Chr: chromosome no. which lncRNA is transcribed. Strand: the strand of chromosome which lncRNA is transcribed; ' + ' is sense strand of chromosome, “-' is antisense strand of chromosome. Relationship: "sense exon overlap": the LncRNA's exon is overlapping a coding transcript exon on the same genomic strand; "sense intron overlap": the LncRNA is overlapping the intron of a coding transcript on the same genomic strand; "antisense exon overlap": the LncRNA is transcribed from the antisense strand and overlapping with a coding transcript; "antisense intron overlap": the LncRNA is transcribed from the antisense strand without sharing overlapping exons;"bidirection": the LncRNA is oriented head to head to a coding transcript within $1000 \mathrm{bp}$; "intergenic": there are no coding transcripts within $30 \mathrm{~kb}$ of the LncRNA; "others": means other LncRNAs. AD13,Control1-3:Normalized Intensity of each sample (log2 transformed)

a

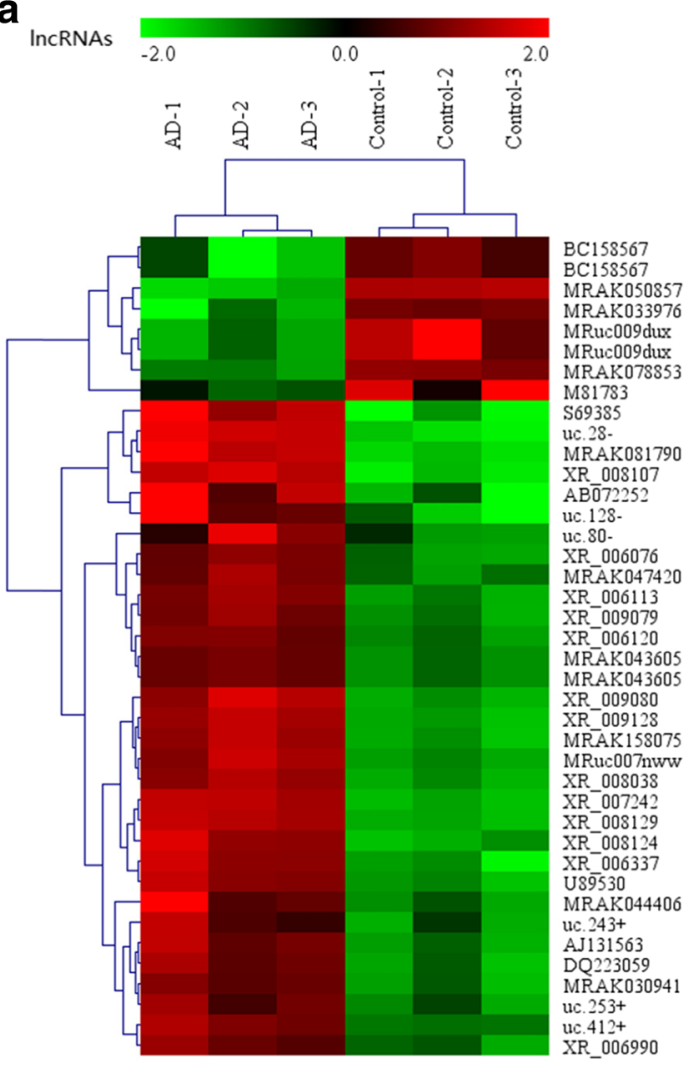

Fig. 2 The heat map and hierarchical clustering of the top 40 differentially expressed lncRNAs (a) and mRNAs (b) between AD and control hippocampal samples. The data are depicted as a data matrix, in which each row represents one lncRNA (mRNA) and each column represents one of the hippocampal samples. The relative lncRNA

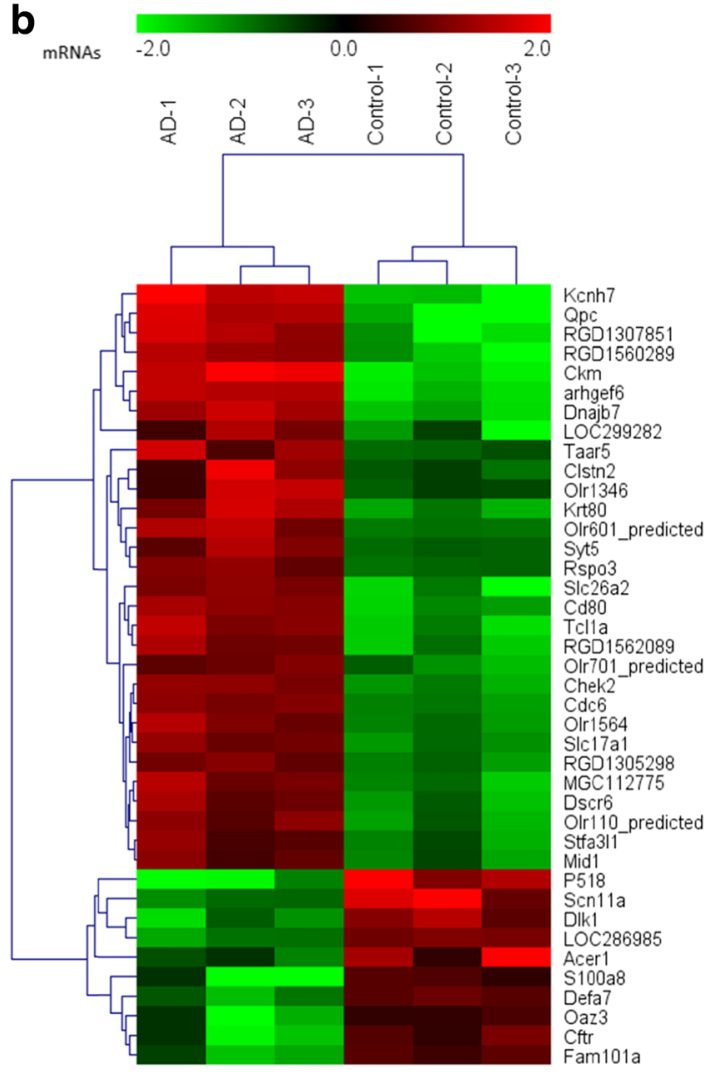

(mRNA) expression is depicted according to the colour scale shown at the top. Red represents high relative expression, and green represents low relative expression; $-2.0,0$ and 2.0 are $\mathrm{FCs}$ in the corresponding spectrum. The magnitude of deviation from the median is represented by the colour saturation 
a

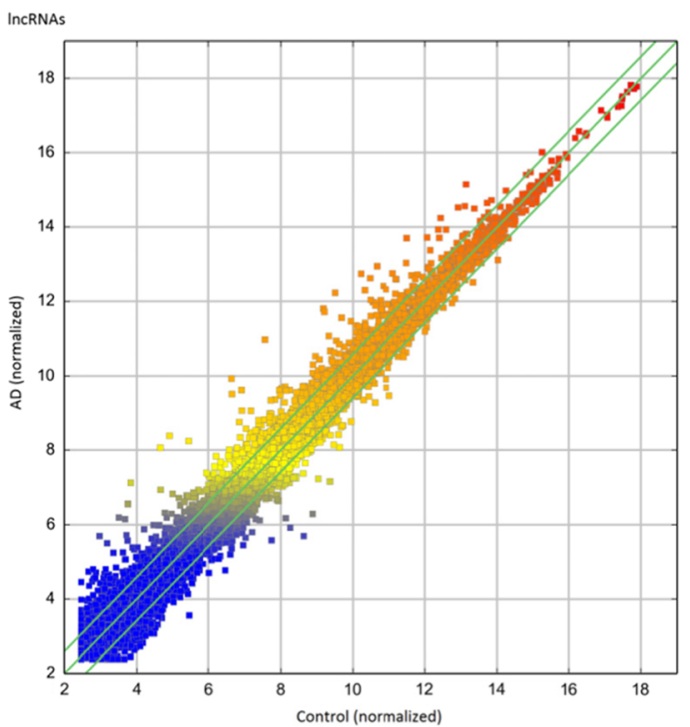

Fig. 3 The scatter plot of IncRNA (a) and mRNA (b) expression variation between the $\mathrm{AD}$ and control hippocampal samples. The values shown on the $\mathrm{x}$-axis and $\mathrm{y}$-axis in the scatter plot are the normalized signal values of each sample ( $\log 2$ scale). The green lines are fold-

differentially expressed lncRNAs are shown in Table 2. Among the dysregulated lncRNA transcripts, S69385 was the most down-regulated, with an FC of 11.25 , whereas MRAK050857 was the most up-regulated, with an FC of 7.65. The variation of IncRNA expression between the $\mathrm{AD}$ and control rats is shown with a scatter plot (Fig. 2a). The clustering analysis revealed the relationships among lncRNA expression patterns in different samples (Fig. 3a).

Using microarray analysis, we identified 311 significantly dysregulated mRNAs in the AD rats: 191 were up-regulated, while 120 were down-regulated ( $\geq 2.0$ fold, $p<0.05$ ). The top 40 most significantly differentially expressed mRNAs are shown in Table 3. The most up-regulated and downregulated mRNA transcripts were Ckm(NM_012530) and P518 (NM_198200), with an FC of 12.08 and 10.05, respectively. The variation of mRNA expression between the AD and control rats is shown with a scatter plot (Fig. 2b). The clustering analysis revealed the relationships among mRNA expression patterns in different samples (Fig. 3b).

\section{Validation of the Microarray Data Using qRT-PCR}

A total of six dysregulated lncRNAs and mRNAs were randomly selected to validate the microarray results using qRTPCR. Consistent with the microarray chip data, the qRT-PCR results demonstrated that the lncRNAs MRAK088596, MRAK081790 and MAPK10 were up-regulated and that BC092582, MRAK050857 and S100A8 were downregulated in the $\mathrm{AD}$ rats compared with the controls (Fig. 4). b

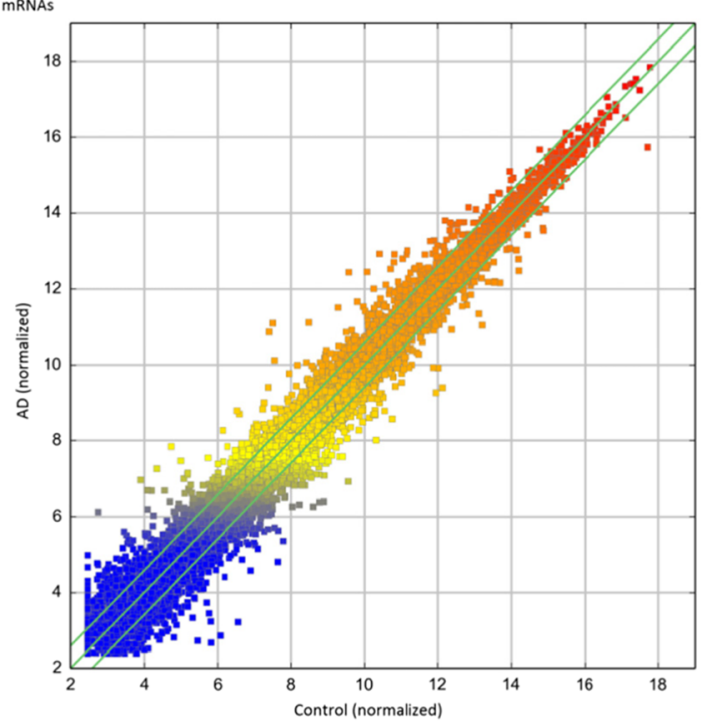

change lines (the default fold-change value given is 2.0). The lncRNAs (mRNAs) above the top green line and below the bottom green line showed an FC $>2.0$ in expression between the 2 compared samples

\section{Gene Enrichment and KEGG Pathway Analyses}

To predict the functions of the IncRNAs, we employed a previously described method [27]. In brief, we first identified and then conducted a functional enrichment analysis of the mRNAs co-expressed with each of the differentially expressed lncRNAs. The enriched functional terms were used as the predicted functional terms for each given lncRNA.

As shown in Fig. 5, the GO analysis indicated that the most enriched GO terms targeted by the mRNAs co-expressed with IncRNAs were endocrine process (ontology: biological process, GO:0050886), extracellular region part (ontology: cellular component, GO:0044421) and neurokinin receptor binding (ontology: molecular function, GO:0031834). Furthermore, the KEGG pathway analysis indicated that the mRNAs co-expressed with lncRNAs were involved in the regulation of antigen processing and presentation, neuroactive ligand-receptor interaction, axon guidance, and the synaptic vesicle cycle, among others. The top 40 KEGG pathways are listed in Fig. 6.

\section{IncRNA-mRNA Network Analysis}

As shown in Fig. 7, the whole co-expression network profile consisted of 454 network nodes and 478 connections among 168 differentially expressed mRNAs and 286 differentially expressed lncRNAs. There were 200 negative and 278 positive interactions within the network. Moreover, our data showed that one mRNA may correlate with 1-66 lncRNAs and that one lncRNA may correlate with 1-2 mRNAs. 
Table 3 Top 40 aberrantly expressed mRNAs in microarray analysis

\begin{tabular}{|c|c|c|c|c|c|c|c|c|c|c|c|}
\hline SeqID & GeneSymbol & $P$ value & $\begin{array}{l}\text { Fold } \\
\text { change }\end{array}$ & Regulation & Chr & AD-1 & AD-2 & AD-3 & Control-1 & Control - 2 & Control - 3 \\
\hline NM_131912 & Kcnh7 & 0.00037 & 11.06140 & Up & 3 & 11.30127 & 10.61836 & 10.70082 & 7.60876 & 7.65469 & 6.95462 \\
\hline NM_001025134 & Qpc & 0.00032 & 10.39597 & Up & 10 & 6.37899 & 5.97278 & 6.02696 & 3.27530 & 2.50436 & 2.46520 \\
\hline NM_001013861 & RGD1307851 & 0.00069 & 8.45568 & Up & 10 & 7.27314 & 6.97233 & 6.68490 & 4.41475 & 3.46865 & 3.80720 \\
\hline NM_001134623 & RGD1560289 & 0.00132 & 7.65156 & Up & 4 & 7.46718 & 7.21876 & 7.13580 & 4.89982 & 4.42654 & 3.68811 \\
\hline NM_012530 & $\mathrm{Ckm}$ & 0.00006 & 12.08045 & Up & 1 & 10.79360 & 11.35903 & 11.15888 & 7.39529 & 7.74974 & 7.38268 \\
\hline NM_001005565 & arhgef6 & 0.00002 & 8.69988 & Up & $\mathrm{X}$ & 7.92966 & 7.82064 & 7.80281 & 4.57567 & 4.98291 & 4.63155 \\
\hline NM_001130510 & Dnajb7 & 0.00009 & 7.37606 & Up & 7 & 12.31645 & 12.68693 & 12.35062 & 9.52755 & 9.82563 & 9.35227 \\
\hline NM_182474 & LOC299282 & 0.03959 & 6.15219 & Up & 6 & 6.26476 & 7.15561 & 6.68019 & 4.53369 & 5.23837 & 2.46520 \\
\hline NM_001009650 & Taar5 & 0.00323 & 3.85177 & Up & 1 & 7.59855 & 6.56191 & 7.18826 & 5.07491 & 5.14253 & 5.29471 \\
\hline NM_134377 & Clstn2 & 0.01116 & 3.67028 & Up & 8 & 10.99065 & 12.38604 & 11.62870 & 9.79077 & 10.00038 & 9.58658 \\
\hline NM_001000520 & Olr1346 & 0.00853 & 3.56797 & Up & 9 & 6.82078 & 7.98940 & 7.87742 & 5.57183 & 5.83954 & 5.77092 \\
\hline NM_001008815 & Krt80 & 0.00069 & 5.82118 & Up & 7 & 12.52671 & 13.29509 & 12.98709 & 10.29545 & 10.68643 & 10.20308 \\
\hline NM_001000512 & Olr601_predicted & 0.00034 & 4.60897 & Up & 3 & 4.79276 & 4.92615 & 4.29177 & 2.42778 & 2.50436 & 2.46520 \\
\hline NM_019350 & Syt5 & 0.00086 & 3.58122 & Up & 1 & 12.20464 & 12.90256 & 12.50704 & 10.63153 & 10.75588 & 10.70548 \\
\hline NM_001100990 & Rspo3 & 0.00012 & 3.52906 & Up & 1 & 9.81657 & 9.94154 & 9.54918 & 7.87261 & 7.97046 & 8.00638 \\
\hline NM_057127 & Slc26a2 & 0.00126 & 5.92500 & Up & 18 & 10.07056 & 10.23371 & 10.05481 & 7.40892 & 8.14971 & 7.09999 \\
\hline NM_012926 & $\mathrm{Cd} 80$ & 0.00021 & 5.62333 & Up & 11 & 7.63253 & 7.42776 & 7.38456 & 4.64968 & 5.24624 & 5.07467 \\
\hline NM_001109601 & Tclla & 0.00082 & 6.24817 & Up & 6 & 11.46388 & 10.90778 & 11.00265 & 8.32631 & 8.94392 & 8.17378 \\
\hline NM_001130502 & RGD1562089 & 0.00125 & 5.29588 & Up & 7 & 12.79245 & 12.27743 & 12.31050 & 9.80832 & 10.54440 & 9.81304 \\
\hline NM_001000627 & Olr701_predicted & 0.00104 & 3.99558 & Up & 3 & 5.37576 & 5.48705 & 5.67957 & 3.90762 & 3.48223 & 3.15731 \\
\hline NM_053677 & Chek2 & 0.00009 & 4.79475 & Up & 12 & 8.86950 & 8.84403 & 8.67135 & 6.52976 & 6.75512 & 6.31564 \\
\hline NM_001108298 & Cde6 & 0.00004 & 4.32074 & $\mathrm{Up}$ & 10 & 6.27822 & 6.10731 & 6.20886 & 4.13715 & 4.21775 & 3.90566 \\
\hline NM_001000045 & Olr1564 & 0.00050 & 4.37034 & Up & 11 & 8.59920 & 8.19315 & 8.01413 & 6.13911 & 6.33668 & 5.94745 \\
\hline NM_133554 & Slc17a1 & 0.00021 & 4.07956 & Up & 17 & 9.61966 & 9.26160 & 9.35299 & 7.23515 & 7.61283 & 7.30103 \\
\hline NM_001134526 & RGD1305298 & 0.00028 & 3.80143 & Up & 6 & 12.70549 & 12.87161 & 12.56917 & 10.78576 & 11.02570 & 10.55518 \\
\hline NM_001044267 & MGC112775 & 0.00171 & 4.73274 & Up & 5 & 11.89504 & 11.26799 & 11.38203 & 9.38440 & 9.60196 & 8.83066 \\
\hline NM_001105892 & Dscr6 & 0.00213 & 4.35798 & Up & 11 & 13.28813 & 12.66876 & 12.81105 & 10.74262 & 11.23262 & 10.42171 \\
\hline NM_001000743 & Olr110_predicted & 0.00148 & 4.29979 & Up & 1 & 8.85370 & 8.41559 & 8.83855 & 6.45820 & 7.04732 & 6.28952 \\
\hline NM_001009177 & Stfa311 & 0.00395 & 3.52549 & Up & 11 & 10.36907 & 9.74567 & 9.85851 & 8.14118 & 8.59783 & 7.78076 \\
\hline NM_022927 & Mid1 & 0.00299 & 3.42529 & Up & $\mathrm{X}$ & 12.30728 & 11.74943 & 11.98512 & 10.15123 & 10.66069 & 9.90122 \\
\hline NM_198200 & P518 & 0.00356 & 10.05736 & Down & 3 & 2.53407 & 3.09987 & 4.04598 & 7.14801 & 6.07090 & 6.45154 \\
\hline NM_019265 & Scn11a & 0.01023 & 6.57096 & Down & 8 & 2.53407 & 2.81928 & 2.83517 & 5.38697 & 6.48818 & 4.46168 \\
\hline NM_053744 & Dlk1 & 0.00305 & 4.86921 & Down & 6 & 7.49082 & 8.48974 & 8.07381 & 10.28931 & 10.66765 & 9.94847 \\
\hline NM_173319 & LOC286985 & 0.00018 & 3.94332 & Down & 1 & 2.53407 & 2.95543 & 2.97305 & 4.73203 & 4.86678 & 4.80198 \\
\hline NM_001106875 & Acer1 & 0.02224 & 3.93238 & Down & Un & 3.39316 & 3.63306 & 2.98687 & 5.32956 & 4.41967 & 6.19006 \\
\hline NM_053822 & S100a8 & 0.02769 & 4.93494 & Down & 2 & 4.79954 & 2.68874 & 2.88047 & 5.87408 & 5.83380 & 5.56997 \\
\hline NM_001033075 & Defa7 & 0.00194 & 3.43660 & Down & 16 & 5.59667 & 4.78587 & 5.36146 & 6.97259 & 7.13337 & 6.98098 \\
\hline NM_001101018 & Oaz3 & 0.02678 & 3.46768 & Down & 2 & 5.80303 & 4.00098 & 4.82002 & 6.60239 & 6.61141 & 6.79215 \\
\hline NM_031506 & $\mathrm{Cftr}$ & 0.01607 & 3.90136 & Down & 4 & 4.62705 & 3.08700 & 3.48477 & 5.67597 & 5.42079 & 5.99400 \\
\hline NM_001109547 & Fam101a & 0.00538 & 3.39621 & Down & 12 & 6.88143 & 5.85283 & 6.07142 & 8.08245 & 7.88576 & 8.12925 \\
\hline
\end{tabular}

\section{Discussion}

Analysing the expression profiles of lncRNAs may provide new insights into our understanding of the aetiology and pathophysiology of AD. In previous studies, D.Y. Lee et al. examined the dysregulated expression of intergenic lncRNAs
(lincRNAs), a significant subgroup of lncRNAs, in a triple transgenic model of AD (3xTg-AD) [16], X. Zhou et al. identified AD-associated lncRNAs based on post-mortem tissue samples of AD patients [14]. Additionally, M. magistri et al. identified several annotated and non-annotated lincRNAs that are differentially expressed in the hippocampus in late-onset 


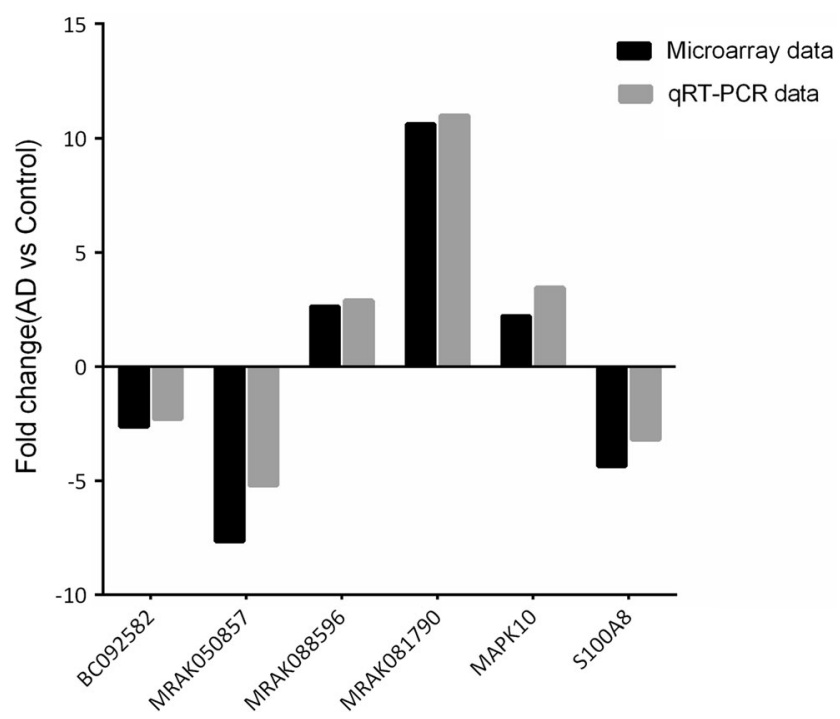

Fig. 4 The differential expression of lncRNAs and mRNAs was validated by quantitative real-time PCR (qRT-PCR). The data showed that the expression levels of the lncRNAs BC092582 and MRAK050857 and the mRNA S100A8 were down-regulated and that the lncRNAs MRAK088596 and MRAK081790 and the mRNA MAPK10 were up-regulated in the hippocampal tissue samples from $\mathrm{AD}$ rats relative to the control rats. The heights of the columns in the chart represent FCs. The qRT-PCR results were consistent with the microarray data

Alzheimer's disease (LOAD) [15]. Despite these previously reported findings, the roles of IncRNAs in $\mathrm{AD}$ remain largely unknown because none of the currently available models recapitulate all aspects of human $\mathrm{AD}$ [28].

As a useful experimental animal model of AD emphasizing the inflammatory component of the disease pathology, the $\mathrm{A} \beta_{1-42}$ infusion model strongly complements the use of transgenic animal models in advancing our understanding of $\mathrm{AD}$ [29]. However, comprehensive studies into the special expression patterns of lncRNAs in an $A \beta_{1-42}$ infusion AD model have not been reported. Thus, to the best of our knowledge, this study is the first to describe hippocampal lncRNAs in an $A \beta_{1-42}$ infusion $\mathrm{AD}$ model and further our understanding of IncRNAs that are associated with the pathogenesis of AD.

In the present study, we identified $315 \operatorname{lncRNAs}$ and 311 mRNAs using a second-generation lncRNA microarray. Of these, 238 lncRNAs and 191 mRNAs were found to be significantly up-regulated in the AD rats compared with the control rats ( $\mathrm{FC} \geq 2.0, p<0.05$ ). In addition, several of the dysregulated lncRNAs and mRNAs, we identified were randomly chosen for qRT-PCR validation, and the results confirmed the microarray analysis findings to some extent. Among the dysregulated lncRNAs, 115 lincRNAs were found. Regarding the previous studies mentioned above, 472 significantly dysregulated mRNAs and 205 lincRNAs were identified in 3xTg-AD mice [16], 64 significantly dysregulated lincRNAs were found in $\mathrm{AD}$ patients [14], and 89 lincRNAs were found to be differentially expressed in the hippocampus of LOAD patients
[15]. Taken together, these findings will likely lead towards a better understanding of the function of dysregulated lncRNAs in the neuropathogenesis of $\mathrm{AD}$.

To predict the potential functions of the differentially expressed lncRNAs identified in this study, GO and KEGG pathway analyses were performed using the coding genes associated with the significantly differentially expressed lncRNAs. GO analysis revealed that these lncRNAs are involved in such biological processes as synaptic transmission regulation, cholinergic regulation, central nervous system neuron differentiation, external stimulus response processes and endocrine processes, all of which are important in learning and memory, as well as the development of AD. KEGG pathway analysis indicated that the genes associated with the dysregulated lncRNAs in the $\mathrm{AD}$ group are involved in the neuroactive ligand-receptor interaction, the renin-angiotensin system, axon guidance, and the PI3K-Akt, MAPK, and mTOR signalling pathways. Among these, the PI3K-Akt, MAPK, and mTOR signalling pathways play important roles in longterm learning and memory, such as in neurocyte nutrition, encoding protein synthesis regulation for memory formation in the hippocampus, and memory production and consolidation [30-32].

Furthermore, of these dysregulated lncRNAs, we identified AJ131563 and MRAK043570, which are associated with the insulin signalling pathway. Growing evidence suggests that the deregulation of insulin signalling in the brain plays an important role in the development of $\mathrm{AD}$, which is involved in numerous molecular pathogeneses, including APP overexpression, $A \beta$ accumulation, tau hyperphosphorylation, neuroinflammation, oxidative stress promotion and synaptic failure $[33,34]$. On the other hand, the IncRNA MRAK043570 is significantly associated with the PI3K-Akt, mTOR, FoxO and AMPK signalling pathways, which are altered in brains with $\mathrm{AD}[35,36]$; in addition, all these pathways are linked to the regulation of autophagy, which is strongly regarded as one of the major pathogenic mechanisms of $\mathrm{AD}[37,38]$. These findings suggest that altered lncRNAs may be involved in AD-associated signalling pathways. However, our knowledge about the potential functions of these dysregulated lncRNAs in the neuropathogenesis of $\mathrm{AD}$ remains limited. Thus, further investigation is of great importance.

In the present study, we also employed an IncRNA-mRNA network analysis to identify interactions between differentially expressed mRNAs and differentially expressed lncRNAs, as previously described $[39,40]$. The co-expression network reported here was constructed based on the 315 differentially expressed lncRNAs and the 311 differentially expressed mRNAs distinguishing the $\mathrm{AD}$ rats from the control rats. Our results showed that a total of $168 \operatorname{lncRNAs}$ and 286 mRNAs were included in the co-expression network, which consisted of 454 network nodes and 478 connections. We also found that S100a8, an mRNA, was correlated with up to 66 


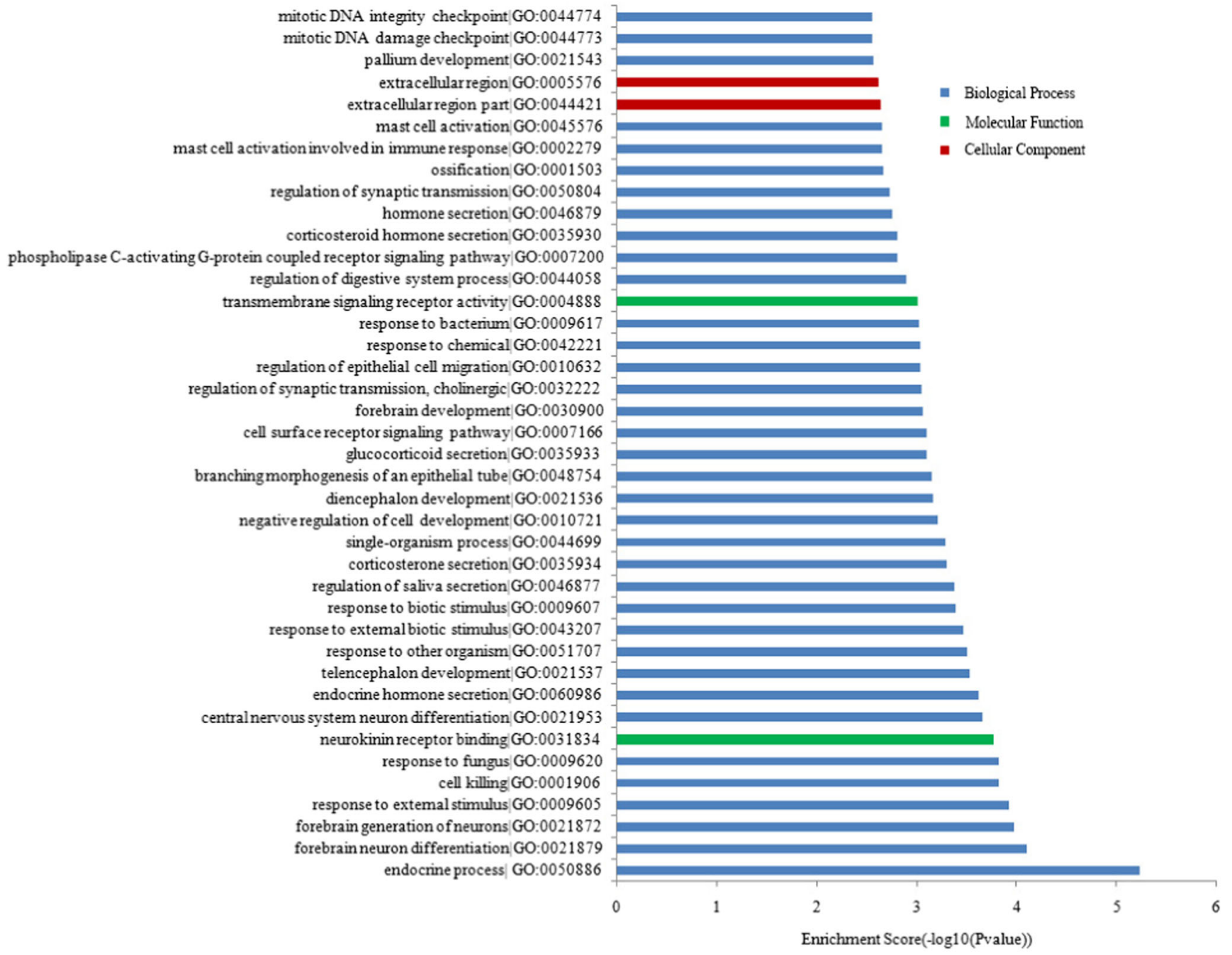

Fig. 5 Top $40 \mathrm{GO}$ terms for the differences in co-expressed lncRNA genes in $\mathrm{AD}$ animals and the controls. The GO enrichment analysis provided a controlled vocabulary to describe the co-expressed genes of

lncRNAs; similarly, Np4 was correlated with 41 lncRNAs, Mcpt9 with 27 lncRNAs, Defa with 24 lncRNAs. Interestingly, a previous study has demonstrated that four mRNAs (S100a8, Np4, Mcpt9, and Defa) were involved in the neuroinflammatory responses in the frontal cortex of ageing female rats [41]. Because neuroinflammatory responses have been implicated as a significant contributor to AD pathogenesis [42], the specific roles of these mRNAs with respect to the neuropathology of AD deserve further investigation. In addition, $\mathrm{S} 100 \mathrm{a} 8$ is a ligand of the receptor for advanced glycation end products, which is a receptor in the immunoglobulin superfamily that also binds other ligands, including advanced glycation end products, the high-mobility group protein $\mathrm{B} 1$ (HMGB1), and $\mathrm{A} \beta$. In addition, $\mathrm{S} 100 \mathrm{~A} 8$ could modulate APP processing towards increased $\beta$-secretase activity and the production of long, more amyloidogenic, $\mathrm{A} \beta$ peptides [43]. the differentially expressed lncRNAs. The ontology covered three domains: biological process, cellular component, and molecular function

We also found that 172 lncRNAs interacted with 5 mRNAs that are mainly involved in the neuroactive ligand-receptor interaction, lysosome and renin-angiotensin system signalling pathways, which have been demonstrated to play important roles in the pathophysiology of AD and could serve as potential therapeutic targets [44-46]. The co-expression network suggests that the inter-regulation of lncRNAs and mRNAs is involved in $\mathrm{AD}$ and warrants further study.

Although altered lncRNAs and mRNAs were identified and their possible roles in the pathophysiology of AD were investigated in this study, several limitations should be acknowledged. First, the analysis was only performed using the hippocampus of AD animals. Global lncRNA and mRNA changes in the blood and cerebral spinal fluid of the same AD model animals should be also determined in further studies to more accurately reflect the pathophysiology of AD. Second, the present study only predicted lncRNA functions 


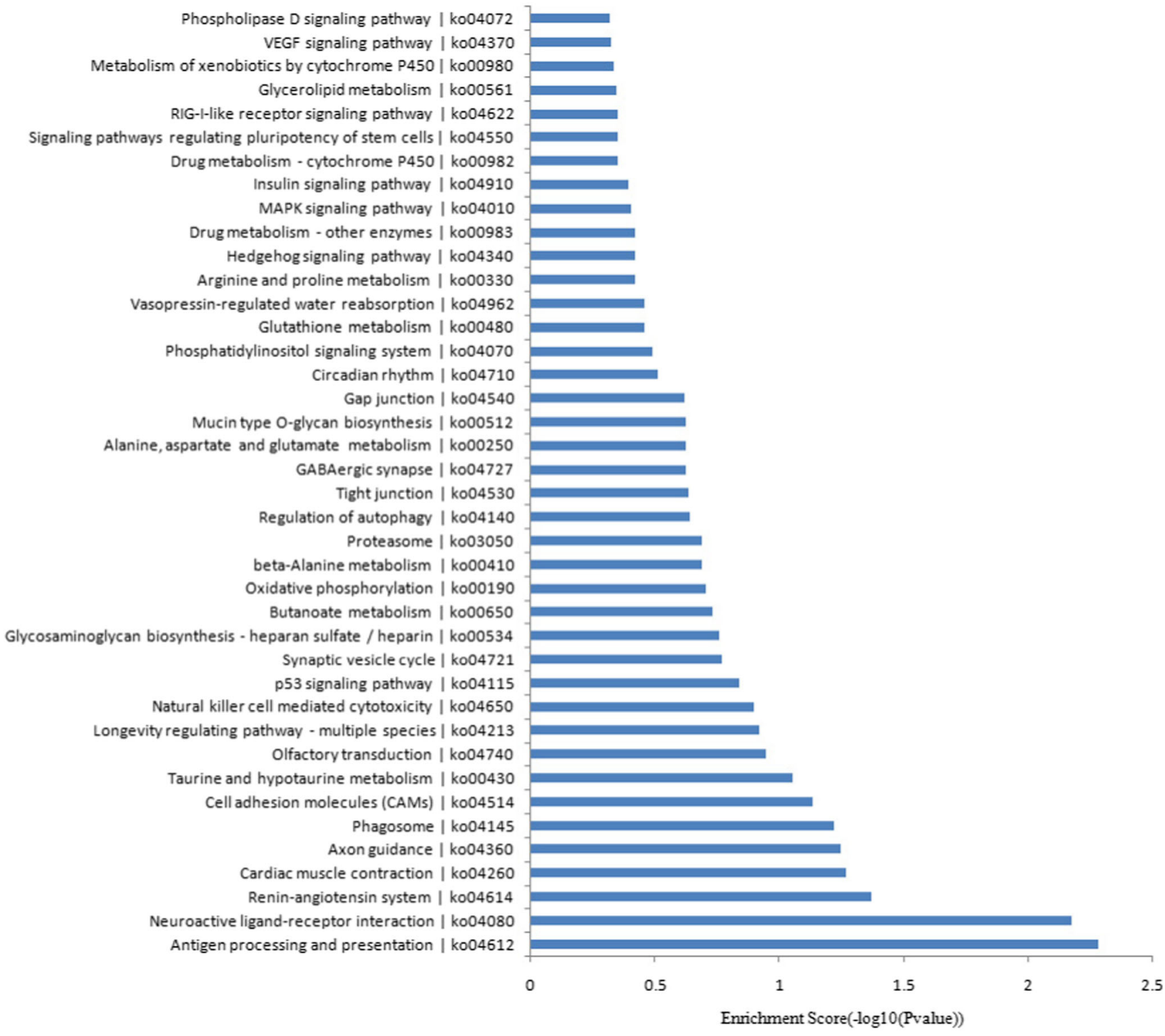

Fig. 6 KEGG pathways analysis. Top 40 pathways for the differences in lncRNA genes co-expressed in AD animals and the controls

through investigating the functional significance of the mRNAs co-expressed with the differentially expressed lncRNAs. Third, gene expression microarrays have a limited dynamic range and lack the ability to identify novel features, such as splice isoforms or fusion transcripts. RNA-seq technology allows the discovery of previously inaccessible complexities in the transcriptome, such as allele-specific expression and novel promoters and isoforms. However, the resulting datasets are large and complex, and their interpretation is not straightforward [47]. Fourth, similar to previous studies $[47,48]$, we also only performed the minimal number of experiments, which may result in an underestimation of the number of altered lncRNAs and mRNAs. Larger sample sizes could achieve more optimal results. Additionally, further research should select from 8 to 10 up-regulated and 8 to 10 down-regulated lncRNAs for microarray validation and include a larger sample size than that in the present study. Future studies that overcome the limitations mentioned above are merited.

In conclusion, the present study uses microarray data to reveal for the first time that the hippocampal expression patterns of $\operatorname{lncRNAs}$ are significantly altered in AD. In addition, the data indicate that aberrantly expressed IncRNAs participate in several specific biological processes and are involved in related pathways that may contribute to the pathogenesis of AD. While these findings provide newfound information regarding the potential role of lncRNAs in $\mathrm{AD}$, further research is required to fully elucidate the detailed molecular mechanisms underlying the action of significantly dysregulated lncRNAs. 


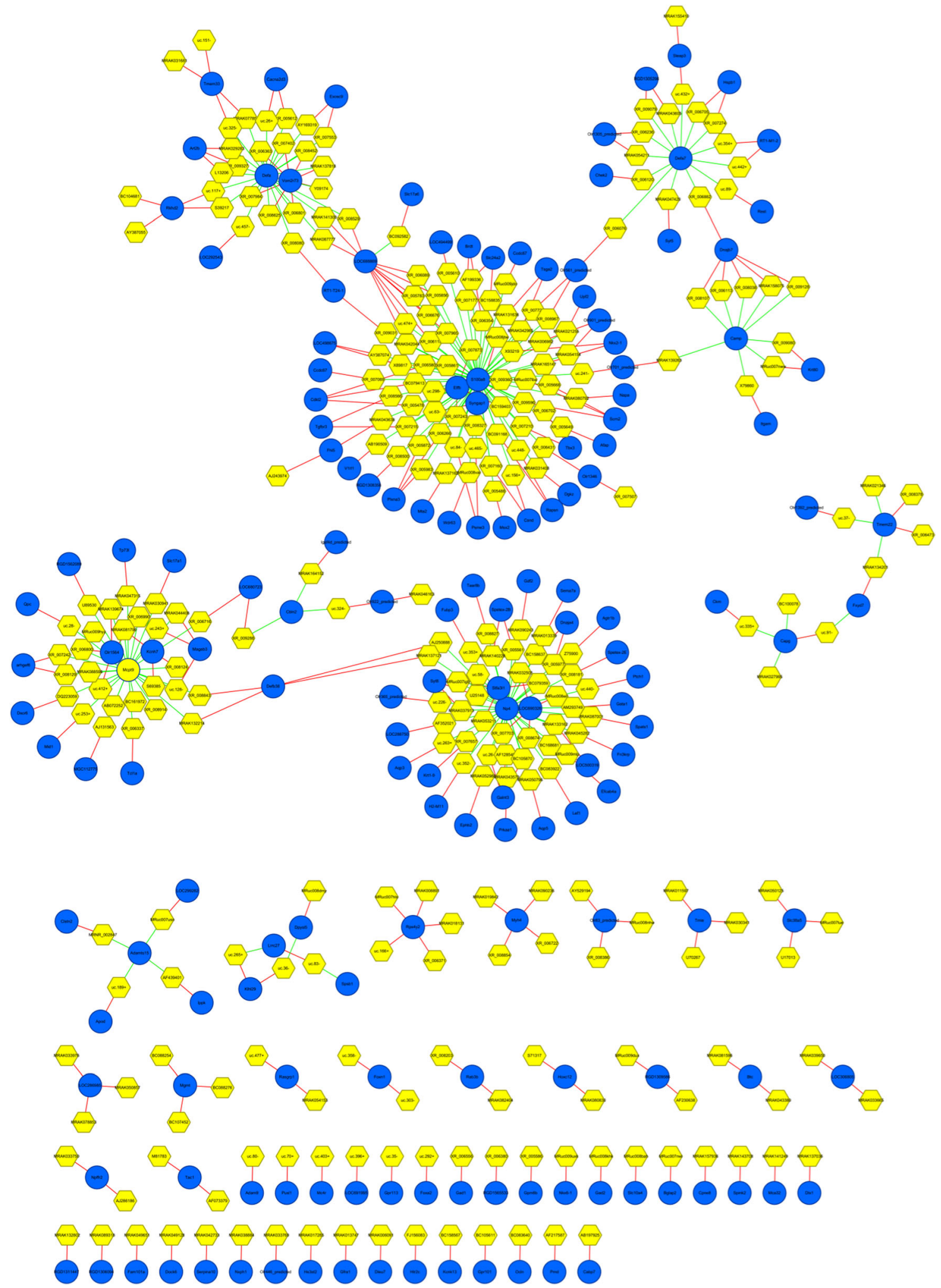

Fig. 7 lncRNA-mRNA-network analysis. Blue nodes represent dysregulated lncRNAs, yellow nodes represent dysregulated mRNAs. The red lines between IncRNAs and mRNAs indicate a negative correlation, while the green lines indicate a positive correlation 
Acknowledgments This study was financially supported by the Young Scientists Fund of the National Natural Science Foundation of China (grant no. 81303074) and the National Natural Science Foundation of China (grant no. 81373705). We also thank Gene Denovo Co. (Guangzhou, People's Republic of China) for technical support.

Author Contributions The work presented here was carried out in collaboration among all authors. Z.W. and W.P. conceived and designed the work and helped to coordinate support and funding. B.Y. and W.P. wrote the manuscript. Z.X., C.S., W.G. and Y.C. performed the animal experiments and helped collect the samples. B.Y. and B.Z. participated in the acquisition, analysis and interpretation of the data. X.X. and Y.W. coworked on associated data collection and analysis. All authors reviewed and approved the final manuscript.

\section{Compliance with Ethical Standards}

Competing Interests The authors declare that they have no competing interests.

Open Access This article is distributed under the terms of the Creative Commons Attribution 4.0 International License (http:// creativecommons.org/licenses/by/4.0/), which permits unrestricted use, distribution, and reproduction in any medium, provided you give appropriate credit to the original author(s) and the source, provide a link to the Creative Commons license, and indicate if changes were made.

\section{References}

1. Xu W, Weissmiller AM, White JA, 2nd, Fang F, Wang X, Wu Y, et al. (2016) Amyloid precursor protein-mediated endocytic pathway disruption induces axonal dysfunction and neurodegeneration. $\mathrm{J}$ Clin Invest 5:1815-1833

2. Alzheimer's Association (2016) 2016 Alzheimer's disease facts and figures. Alzheimers Dement 12:459-509

3. Huang Y, Mucke L (2012) Alzheimer mechanisms and therapeutic strategies. Cell 148:1204-1222

4. Hendrix JA, Bateman RJ, Brashear HR, Duggan C, Carrill MC, Bain LJ, et al. (2016) Challenges, solutions, and recommendations for Alzheimer's disease combination therapy. Alzheimers Dement 12:623-630

5. Mercer TR, Dinger ME, Mattick JS (2009) Long non-coding RNAs: insights into functions. Nat Rev Genet 10:155-159

6. Quinn JJ, Chang HY (2016) Unique features of long non-coding RNA biogenesis and function. Nat Rev Genet 17:47-62

7. Wang WT, Sun YM, Huang W, He B, Zhao YN, Chen YQ (2016) Genome-wide long non-coding RNA analysis identified circulating LncRNAs as novel non-invasive diagnostic biomarkers for gynecological disease. Sci Rep 6:23343

8. Yuan J, Yue H, Zhang M, Luo J, Liu L, Wu W, et al. (2016) Transcriptional profiling analysis and functional prediction of long noncoding RNAs in cancer. Oncotarget 7:8131-8142

9. Sunwoo JS, Lee ST, Im W, Lee M, Byun JI, Jung KH, et al. Altered expression of the long noncoding RNA NEAT1 in Huntington's disease. Mol Neurobiol 2016.
10. Faghihi MA, Modarresi F, Khalil AM, Wood DE, Sahagan BG, Morgan TE, et al. (2008) Expression of a noncoding RNA is elevated in Alzheimer's disease and drives rapid feed-forward regulation of beta-secretase. Nat Med 14:723-730

11. Ciarlo E, Massone S, Penna I, Nizzari M, Gigoni A, Dieci G, et al. (2013) An intronic ncRNA-dependent regulation of SORL1 expression affecting abeta formation is upregulated in post-mortem Alzheimer's disease brain samples. Dis Model Mech 6:424-433

12. Mus E, Hof PR, Tiedge H (2007) Dendritic BC200 RNA in aging and in Alzheimer's disease. Proc Natl Acad Sci U S A 104:1067910684

13. Massone S, Vassallo I, Fiorino G, Castelnuovo M, Barbieri F, Borghi R, et al. (2011) 17A, a novel non-coding RNA, regulates GABA B alternative splicing and signaling in response to inflammatory stimuli and in Alzheimer disease. Neurobiol Dis 41:308317

14. Zhou X, Xu J (2015) Identification of Alzheimer's diseaseassociated long noncoding RNAs. Neurobiol Aging 36:2925-2931

15. Magistri M, Velmeshev D, Makhmutova M, Faghihi MA (2015) Transcriptomics profiling of Alzheimer's disease reveal neurovascular defects, altered amyloid-beta homeostasis, and deregulated expression of long noncoding RNAs. J Alzheimers Dis 48:647-665

16. Lee DY, Moon J, Lee ST, Jung KH, Park DK, Yoo JS, et al. (2015) Distinct expression of long non-coding RNAs in an Alzheimer's disease model. J Alzheimers Dis 45:837-849

17. Lau P, Frigerio CS, De Strooper B (2014) Variance in the identification of microRNAs deregulated in Alzheimer's disease and possible role of lincRNAs in the pathology: the need of larger datasets. Ageing Res Rev 17:43-53

18. Mufson EJ, Mahady L, Waters D, Counts SE, Perez SE, DeKosky ST, et al. (2015) Hippocampal plasticity during the progression of Alzheimer's disease. Neuroscience 309:51-67

19. Mu Y, Gage FH (2011) Adult hippocampal neurogenesis and its role in Alzheimer's disease. Mol Neurodegener 6:85

20. Zhong B, Wang Z, He M (2010) Effect of Naoling decoction on the expression of APP in hippocampal CA3 region in rats with synthetic Alzheimer's disease. J Cent South Univ (Med Sci) 35:431-437

21. Xing Z, Xia Z, Peng W, Li J, Zhang C, Fu C, et al. (2016) Xuefu Zhuyu decoction, a traditional Chinese medicine, provides neuroprotection in a rat model of traumatic brain injury via an antiinflammatory pathway. Sci Rep 6:20040

22. Zou Y, Li C, Shu F, Tian Z, Xu W, Xu H, et al. (2015) lncRNA expression signatures in periodontitis revealed by microarray: the potential role of IncRNAs in periodontitis pathogenesis. J Cell Biochem 116:640-647

23. Gebhardt FM, Scott HA, Dodd PR (2010) Housekeepers for accurate transcript expression analysis in Alzheimer's disease autopsy brain tissue. Alzheimers Dement 6:465-474

24. Chen R, Liu L, Xiao M, Wang F, Lin X (2016) Microarray expression profile analysis of long noncoding RNAs in premature brain injury: a novel point of view. Neuroscience 319:123-133

25. Zhao Z, Bai J, Wu A, Wang Y, Zhang J, Wang Z, et al. (2015) CoLncRNA: investigating the lncRNA combinatorial effects in GO annotations and KEGG pathways based on human RNA-seq data. Database (Oxford) 2015

26. Pujana MA, Han JD, Starita LM, Stevens KN, Tewari M, Ahn JS, et al. (2007) Network modeling links breast cancer susceptibility and centrosome dysfunction. Nat Genet 39:1338-1349

27. Guttman M, Rinn JL (2012) Modular regulatory principles of large non-coding RNAs. Nature 482:339-346 
28. Van Dam D, De Deyn PP (2011) Animal models in the drug discovery pipeline for Alzheimer's disease. Br J Pharmacol 164:12851300

29. McLarnon JG, Ryu JK (2008) Relevance of abeta1-42 intrahippocampal injection as an animal model of inflamed Alzheimer's disease brain. Curr Alzheimer Res 5:475-480

30. Shu Y, Zhang H, Kang T, Zhang JJ, Yang Y, Liu H, et al. (2013) $\mathrm{PI} 3 \mathrm{~K} /$ Akt signal pathway involved in the cognitive impairment caused by chronic cerebral hypoperfusion in rats. PLoS One 8: e81901

31. Shobe J, Philips GT, Carew TJ (2016) Transforming growth factor beta recruits persistent MAPK signaling to regulate long-term memory consolidation in Aplysia californica. Learn Mem 23: $182-188$

32. Bekinschtein P, Katche C, Slipczuk LN, Igaz LM, Cammarota M, Izquierdo I, et al. (2007) mTOR signaling in the hippocampus is necessary for memory formation. Neurobiol Learn Mem 87:303-307

33. Bedse G, Di Domenico F, Serviddio G, Cassano T (2015) Aberrant insulin signaling in Alzheimer's disease: current knowledge. Front Neurosci 9:204

34. Chen Y, Deng Y, Zhang B, Gong CX (2014) Deregulation of brain insulin signaling in Alzheimer's disease. Neurosci Bull 30:282-294

35. Godoy JA, Rios JA, Zolezzi JM, Braidy N, Inestrosa NC (2014) Signaling pathway cross talk in Alzheimer's disease. Cell Commun Signal 12:23

36. Manolopoulos KN, Klotz LO, Korsten P, Bornstein SR, Barthel A (2010) Linking Alzheimer's disease to insulin resistance: the FoxO response to oxidative stress. Mol Psychiatry 15:1046-1052

37. Nixon RA (2013) The role of autophagy in neurodegenerative disease. Nat Med 19:983-997

38. Yoon SY, Kim DH (2016) Alzheimer's disease genes and autophagy. Brain Res 1649(Pt B):201-209

39. Xu J, Zhang F, Gao C, Ma X, Peng X, Kong D, et al. Microarray analysis of lncRNA and mRNA expression profiles in patients with neuromyelitis optica. Mol Neurobiol 2016.
40. Dong R, Jia D, Xue P, Cui X, Li K, Zheng S, et al. (2014) Genomewide analysis of long noncoding RNA (lncRNA) expression in hepatoblastoma tissues. PLoS One 9:e85599

41. Sarvari M, Hrabovszky E, Kallo I, Solymosi N, Toth K, Liko I, et al. (2011) Estrogens regulate neuroinflammatory genes via estrogen receptors alpha and beta in the frontal cortex of middle-aged female rats. J Neuroinflammation 8:82

42. Heneka MT, Carson MJ, Khoury JE, Landreth GE, Brosseron F, Feinstein DL, et al. (2015) Neuroinflammation in Alzheimer's disease. Lancet Neurol 14:388-405

43. Lodeiro M, Puerta E, Ismail MA, Rodriguez-Rodriguez P, Ronnback A, Codita A, et al. Aggregation of the Inflammatory S100A8 precedes abeta plaque formation in transgenic APP Mice: positive feedback for S100 A8 and abeta productions. J Gerontol A Biol Sci Med Sci 2016.

44. Coffey EE, Beckel JM, Laties AM, Mitchell CH (2014) Lysosomal alkalization and dysfunction in human fibroblasts with the Alzheimer's disease-linked presenilin 1 A246E mutation can be reversed with cAMP. Neuroscience 263:111124

45. Wharton W, Goldstein FC, Zhao L, Steenland K, Levey AI, Hajjar I (2015) Modulation of renin-angiotensin system may slow conversion from mild cognitive impairment to Alzheimer's disease. J Am Geriatr Soc 63:1749-1756

46. Gu C, Shen T (2014) cDNA microarray and bioinformatic analysis for the identification of key genes in Alzheimer's disease. Int J Mol Med 33:457-461

47. Shen X, Xie B, Ma Z, Yu W, Wang W, Xu D, et al. (2015) Identification of novel long non-coding RNAs in triple-negative breast cancer. Oncotarget 6:21730-21739

48. Liu Q, Ma J, Yu Z, Liu H, Chen C, Li W (2016) Distinct hippocampal expression profiles of lncRNAs in rats exhibiting a PTSDlike syndrome. Mol Neurobiol 53:2161-2168 